\title{
Transcriptome Profile of the Asian Giant Hornet (Vespa mandarinia) Using Illumina HiSeq 4000 Sequencing: De Novo Assembly, Functional Annotation, and Discovery of SSR Markers
}

\author{
Bharat Bhusan Patnaik, ${ }^{1,2}$ So Young Park, ${ }^{1}$ Se Won Kang, ${ }^{1}$ \\ Hee-Ju Hwang, ${ }^{1}$ Tae Hun Wang, ${ }^{1}$ Eun Bi Park, ${ }^{1}$ Jong Min Chung, ${ }^{1}$ Dae Kwon Song, \\ Changmu Kim, ${ }^{3}$ Soonok Kim, ${ }^{3}$ Jae Bong Lee, ${ }^{4}$ Heon Cheon Jeong, ${ }^{5}$ Hong Seog Park, \\ Yeon Soo Han, ${ }^{7}$ and Yong Seok Lee ${ }^{1}$ \\ ${ }^{1}$ Department of Life Science and Biotechnology, College of Natural Sciences, Soonchunhyang University, \\ 22 Soonchunhyangro, Shinchang-myeon, Asan, Chungchungnam-do 31538, Republic of Korea \\ ${ }^{2}$ Trident School of Biotech Sciences, Trident Academy of Creative Technology (TACT), Chandrasekharpur, Bhubaneswar, \\ Odisha 751024, India \\ ${ }^{3}$ National Institute of Biological Resources, 42 Hwangyeong-ro, Seo-gu, Incheon 22689, Republic of Korea \\ ${ }^{4}$ Korea Zoonosis Research Institute (KOZRI), Chonbuk National Institute, 820-120 Hana-ro, Iksan, \\ Jeollabuk-do 54528, Republic of Korea \\ ${ }^{5}$ Hampyeong County Insect Institute, Hampyeong County Agricultural Technology Center, 90 Hakgyohwasan-gil, \\ Hakgyo-myeon, Jeollanam-do 57158, Republic of Korea \\ ${ }^{6}$ Research Institute, GnC BIO Co., Ltd., 621-6 Banseok-dong, Yuseong-gu, Daejeon 34069, Republic of Korea \\ ${ }^{7}$ College of Agriculture and Life Sciences, Chonnam National University, 77 Yongbong-ro, Buk-gu, Gwangju 61186, Republic of Korea
}

Correspondence should be addressed to Yong Seok Lee; yslee@sch.ac.kr

Received 29 October 2015; Accepted 20 December 2015

Academic Editor: Elena Pasyukova

Copyright (C) 2016 Bharat Bhusan Patnaik et al. This is an open access article distributed under the Creative Commons Attribution License, which permits unrestricted use, distribution, and reproduction in any medium, provided the original work is properly cited.

Vespa mandarinia found in the forests of East Asia, including Korea, occupies the highest rank in the arthropod food web within its geographical range. It serves as a source of nutrition in the form of Vespa amino acid mixture and is listed as a threatened species, although no conservation measures have been implemented. Here, we performed de novo assembly of the V. mandarinia transcriptome by Illumina HiSeq 4000 sequencing. Over 60 million raw reads and 59,184,811 clean reads were obtained. After assembly, a total of 66,837 unigenes were clustered, 40,887, 44,455, and 22,390 of which showed homologous matches against the PANM, Unigene, and KOG databases, respectively. A total of 15,675 unigenes were assigned to Gene Ontology terms, and 5,132 unigenes were mapped to $115 \mathrm{KEGG}$ pathways. The zinc finger domain (C2H2-like), serine/threonine/dual specificity protein kinase domain, and RNA recognition motif domain were among the top InterProScan domains predicted for $V$. mandarinia sequences. Among the unigenes, we identified 534,922 cDNA simple sequence repeats as potential markers. This is the first transcriptomic analysis of the wasp V. mandarinia using Illumina HiSeq 4000. The obtained datasets should promote the search for new genes to understand the physiological attributes of this wasp.

\section{Introduction}

Vespa mandarinia, the Asian giant hornet, is among the largest eusocial wasps globally and is found in Korea, Japan,
Taiwan, and China, as well as the Indian subcontinent. These wasps prefer dense woodlands and mountains, where they build their colonies and prey on honeybees, larger insects, other wasps, and hornets $[1,2]$. They can cause serious 
damage to apiaries, with a group of 10-20 V. mandarinia workers being reported to be able to destroy a population of 10,000 to 30,000 bees in a colony [3]. As an apex predator, they have no natural enemies (except for the endoparasitoid Xenos moutoni), and humans pose the biggest threat to them due to their economic value as a source of food (served as hornet sashimi). Habitat destruction due to deforestation has placed this species under threat of extinction, which is exacerbated by the lack of proactive conservation initiatives.

The significant economic value of this species is associated with a substance called Vespa amino acid mixture (VAAM), which has been shown to increase swimming endurance and to decrease lactate and increase glucose concentrations after swimming in mice. This substance is sold as a nutritional supplement to improve athletic performance [4]. One study demonstrated that, with regular exercise, VAAM supplements could increase aerobic fitness while decreasing intra-abdominal fat in humans [5]. Additionally, $V$. mandarinia venom is potent enough to cause the skin to hemorrhage and potentially to cause organ failure [6]. The venom is rich in bioactive peptides and high-molecular weight proteins, such as enzymes, allergens, and toxins $[7,8]$.

Despite the economic prospects of this species and the need to conserve it in its natural habitat, very little information about the sequence and function of its genes has been revealed. Currently, the NCBI resource for $V$. mandarinia contains only the mitochondrial protein sequences, silk proteins 1 to 5, prepromastoparan, immune responsive protein 30, 14-3-3 zeta, ecdysone receptor, period beta, vespakinin, and mastoparan peptide sequences. Both vespakinin (12 amino acids) and mastoparan (14 amino acids) peptides, isolated from $V$. mandarinia venom, can cause cell degranulation $[9,10]$. This limited genetic information is insufficient for conducting a high-throughput functional analysis to unravel the physiological processes associated with the feeding, reproduction, and other behaviors of this wasp. Information on the whole genome sequence of this species would reveal rare gene transcripts, new regulatory elements, alternative splicing, and microsatellites, which would facilitate research on the function of targeted genes.

Whole DNA and RNA sequencing strategies have been highly successful in addressing the genomic challenges in eusocial insects. In particular, transcriptome-wide studies have provided insights into the caste system, and the phenotypic plasticity of the genome has been studied in the Western honey bee, Apis mellifera [11]; bumble bee, Bombus terrestris [12]; stingless bee, Melipona quadrifasciata [13]; and tropical paper wasp, Polistes canadensis [14], using conventional and high-throughput sequencing technologies. Furthermore, study in the field of comparative sociogenomics has characterized candidate gene transcripts potentially responsible for behavioral patterns of eusocial insects across lineages [15]. Microarray and digital gene expression signature techniques have also documented differentially expressed genes involved in processes favoring caste-based structures (brain, legs, and ovaries), promoting our understanding of immuneregulatory, stress-signaling, and reproduction pathways $[16$, 17]. Next-generation sequencing (NGS) technologies and the rapid development of high-throughput platforms have raised the bar regarding the sequencing of nonmodel organisms. These technologies offer a collated and comprehensive output for discovering novel transcripts for functional studies and molecular marker development [18]. Illumina NGS platformbased transcriptomic analysis is reliable for the detection and quantification of active gene transcripts, including novel ones. Illumina HiSeq 2000/2500 platforms have been used for the whole-transcriptome analysis of Polistes canadensis [14] and Cotesia chilonis [19], venom gland transcriptomic analysis of the Brazilian ant species Tetramorium bicarinatum [20], and developmental transcriptomic analysis of Megalopta genalis [21], among others.

In this study, we isolated RNA from queens of the Asian giant hornet, V. mandarinia, constructed a cDNA library, and conducted whole-transcriptome sequencing using the latest Illumina platform, HiSeq 4000. The sequencer-derived raw reads were preprocessed to quality reads and subsequently processed to contig assembly and unigene clusters using the Trinity de novo assembler. For the first time, we report identification of large-scale genomic sequence information and the discovery of microsatellite markers from $V$. mandarinia.

\section{Materials and Methods}

2.1. Biological Samples and RNA Extraction. Specimens of Vespa mandarinia were collected (on 16 June 2015) at Geomdan Mountain, Sinjang-dong, Hanam-si, Gyeonggido Province, South Korea. The sampling for experiments was conducted in accordance with the International Guiding Principles for Biomedical Research Involving Animals (1985) (http://www.ncbi.nlm.nih.gov/books/NBK25438/). Total RNA was isolated from the queens ( $n=10$; whole-body samples) using TRIzol reagent (Invitrogen) following the manufacturer's instructions. The quality of the processed RNA samples was checked using a NanoDrop 2000 spectrophotometer (Thermo, USA) and by running samples on an agarose gel.

\subsection{Construction of cDNA Library and Illumina HiSeq 4000} Sequencing. RNA samples were sent to $\mathrm{GnC}$ Bio Company (Yuseong-gu, Daejeon, South Korea) for mRNA-seq library construction and sequencing using Illumina HiSeq 4000 (Illumina, Inc., San Diego, CA, USA) next-generation platform technology. For mRNA-seq library construction from total RNA, an mRNA-seq sample preparation kit (Illumina Inc., San Diego, CA, USA) was used in accordance with the manufacturer's instructions. Briefly, the total RNA was treated with DNase I and poly-T oligo attached magnetic beads to elute poly- $\left(\mathrm{A}^{+}\right)$mRNA. The purified mRNA was fragmented using a DNA fragmentation kit (Ambion, Austin, TX, USA) prior to cDNA synthesis. The cleaved mRNA fragments were primed using random-hexamer primers and reverse transcriptase (Invitrogen, Carlsbad, CA, USA) to synthesize first-strand cDNA. The synthesis of second-strand cDNA was accomplished using RNase $\mathrm{H}$ (Invitrogen) and DNA polymerase I (New England BioLabs, Ipswich, MA, USA). Subsequently, end-repair of double-stranded cDNA was performed using T4 DNA polymerase, the Klenow fragment, and T4 polynucleotide kinase (New England BioLabs). 
The end-repaired cDNA was ligated to Illumina paired-end (PE) adapter Oligo-mix with T4 DNA ligase (New England BioLabs) at room temperature for $15 \mathrm{~min}$. Suitable fragments $(200 \pm 25 \mathrm{bp})$ were then sequenced in a PE (paired-end) pattern on an Illumina sequencing machine. The sequencing data received from the sequencer were transformed by base calling into raw reads and stored in fastq format.

\subsection{De Novo Assembly of Illumina Sequencing Results.} Before performing transcriptome assembly, the raw reads were filtered to remove adapter-only sequences (number of nucleotides of recognized adapter $\leq 13$, and number of nucleotides excluding the adapter $\leq 35$ ), reads containing unknown nucleotides at a rate of over 5\%, and low-quality reads (Phred quality score $<20$ ), which could affect the efficiency of assembly. For this purpose, we used the commandline tool Cutadapt (http://code.google.com/p/cutadapt/) with default parameters. The preprocessed clean reads were then used for de novo assembly using the short read assembling program Trinity [22], with a $k$-mer size of 25 . The Trinity assembler combined reads (with default options and minimum allowed length of $200 \mathrm{bp}$ ) to form longer overlapping fragments without gaps, forming contigs. Using the TIGR gene indices clustering tool (TGICL) [23], contigs were assembled into unigenes (having $94 \%$ identity, 30 bp overlap). To examine the integrity of the assembly, the unigenes were used as queries in BLASTX against the mitochondrial genome of $V$. mandarinia (GenBank accession KR059904.1).

2.4. Annotation of Unigenes. All of the unigene transcripts were used as queries in searches (BLASTX; E-value $<10^{-5}$ ) against protein databases such as PANM (http://malacol .or.kr/blast/) [24], Unigene, Eukaryotic Clusters of Orthologous Groups (KOG), Gene Ontology (GO), and Kyoto Encyclopedia of Genes and Genomes (KEGG) [25] to determine the number of significant hits to known protein sequences in these databases. The numbers of unigene hits to PANM, Unigene, and KOG were visualized using a three-way Venn diagram plot constructed using Venny 2.0 ( http://bioinfogp.cnb .csic.es/tools/venny/). BLAST2GO software [26] was used to ascribe GO terms to the unigenes assigned to the categories of biological processes, molecular functions, and cellular components. To identify the most prominent protein domains in the unigene sequences, InterProScan searches were performed remotely from BLAST2GO on the InterProEBI server. The BLAST2GO program can be used to find the top hits for unigene sequences using the BLASTX algorithm, assigning InterPro domains, retrieving all available GO terms for each sequence, and mapping for KEGG pathways. A complete schematic representation of transcriptome assembly workflow for Vespa mandarinia is shown in Supplementary Figure 1 in Supplementary Material available online at http://dx.doi.org/10.1155/2016/4169587.

2.5. Simple Sequence Repeat Marker Discovery. The microsatellite identification tool (MISA; http://pgrc.ipk-gatersleben.de/misa/) was used to localize the microsatellites in the unigene sequences of $V$. mandarinia. All types of simple sequence repeat (SSR) were identified, from mononucleotide to decanucleotide repeats, including compound SSRs (with more than one type of repeat unit). The selection criteria for the SSRs were as follows: the minimum repeat unit was 10 for mononucleotides 3 for dinucleotides to decanucleotides. Mononucleotide repeats were not considered for the SSR analysis because of the possibility of problems with the Illumina homopolymer sequencing associated with this technology.

\section{Results and Discussion}

3.1. Illumina HiSeq 4000 Sequencing and V. mandarinia Transcriptome. Illumina NGS platforms have been shown to be suitable for a variety of sequencing applications, including RNA sequencing (RNA-seq), metagenomics, and nucleotide-based marker detection. In RNA-seq applications, Illumina is the preferred choice for de novo mapping of transcripts in nonmodel organisms [27, 28]. With HiSeq 4000 instrument, Illumina takes advantage of patterned flow technology for enhanced output of sequence reads/lane, with $1.5 \mathrm{~Tb}$ PE150 reads in 3.5 days [29]. Using the HiSeq 4000 sequencing platform, we obtained a global overview of the $V$. mandarinia (queen) transcriptome, with over 60 million raw sequences of 9,169,196,254 bases (Table 1). After stringent filtration, 59,184,811 quality reads (Q20 percentage of $99 \%$ ) were obtained with an accumulated length of $8,297,028,222$ bases, representing $97.47 \%$ of the raw reads. A summary of the raw sequence processing based on quality parameters is shown in Supplementary Table 1. The adapter trimming analysis suggested that $1.82 \%$ of raw sequence bases were discarded, generating $98.18 \%$ reads with an average length of $148.3 \mathrm{bp}$. The sequence data of $V$. mandarinia were deposited in the NCBI sequence read archive (SRA) under accession number SRP064879. All data (transcripts, annotations, and reads) related to the $V$. mandarinia transcriptome are publicly hosted on the local submission webpage (http://bioinfo.sch.ac.kr/submission/). The release date of the information (16 October 2016) is one year after the date of submission (16 October 2015).

The quality reads were assembled into contigs using the Trinity short read assembly program. A total of 147,400 contigs with a mean length of 1,230.9 and an N50 length (contig length such that equally long or longer contigs constitute half of the total assembly length) of 2,578 bp were derived from the de novo assembly process. The largest contig assembled was 30,772 bp, and 75,428 contigs were over 500 bp in length. Among the total of 147,400 contigs assembled, 72,077 contigs (48.9\%) had lengths varying between 200 and 500 bp, 27,690 (18.79\%) between 501 and 1,000 bp, 11,606 (7.87\%) between 1,001 and $1,500 \mathrm{bp}$, and $8,873(6.2 \%)$ between 1,501 and $2,000 \mathrm{bp}$ and $27,154(18.42 \%)$ contigs more than 2,001 bp in length (Supplementary Figure 2(A)). Furthermore, a total of 66,837 unigenes were obtained from the clustering of contigs having a mean length and an N50 length of 1,431.2 and $3,112 \mathrm{bp}$, respectively. The length of the unigenes varied from 224 to $52,946 \mathrm{bp}$. Among the 66,837 unigenes clustered from the assembly, 30,668 (45.88\%) unigenes were 224 to $500 \mathrm{bp}$ in 
TABLE 1: Summary statistics of the transcription assembly for Vespa mandarinia.

\begin{tabular}{lc}
\hline Total number of raw reads & \\
Number of sequences & $60,723,154$ \\
Number of bases & $9,169,196,254$ \\
Total number of clean reads & \\
Number of sequences & $59,184,811$ \\
Number of bases & $8,297,028,222$ \\
Mean length (bp) & 140.2 \\
N50 length (bp) & 151 \\
GC \% & 37.93 \\
High-quality reads (\%) & 2.53 (sequences), 9.51 (bases) \\
Number of reads discarded (\%) & \\
Contig information & 147,400 \\
Total number of contig & $181,439,800$ \\
Number of bases & $1,230.9$ \\
Mean length of contig (bp) & 2,578 \\
N50 length of contig (bp) & 35.50 \\
GC \% of contig & 30,772 \\
Largest contig (bp) & 75,428 \\
Number of large contigs $(\geq 500 \mathrm{bp})$ & \\
Unigene information & 66,837 \\
Total number of unigenes & $95,657,681$ \\
Number of bases & $1,431.2$ \\
Mean length of unigene (bp) & 3,112 \\
N50 length of unigene (bp) & 35.26 \\
GC \% of unigene & $224-52,946$ \\
Length ranges (bp) & \\
\hline
\end{tabular}

length, 12,122 (18.14\%) were 501-1,000 bp, 5,004 (7.49\%) were 1,001 to $1,500 \mathrm{bp}, 3,954(5.92 \%)$ were 1,501 to $2,000 \mathrm{bp}$, and $15,089(22.58 \%)$ unigenes were $>2,000$ bp in length (Supplementary Figure 2(B)). The mean length of unigenes obtained in this study was found to be significantly greater than that for the transcriptome assemblies of nonmodel species reported elsewhere $[16,30,31]$. We mapped the assembled unigenes against the 13-protein coding genes of $V$. mandarinia mitochondria using BLASTN at a cutoff $E$-value of $1 E-5$ to clarify the completeness of the Trinity assembly. We found that the mitochondrial-protein coding genes were covered $(\sim 100 \%)$ by only three assembled unigenes or 0.23 unigene transcripts per gene (Table 2), illustrating the integrity of the assembly. The gene NADH dehydrogenase subunit 5 (ND5) alone was mapped within $V$. mandarinia unigene 01523 (Vm_Uni_01523), whereas seven and five mitochondrial genes were mapped within Vm_Uni_08557 and Vm_Uni_10664, respectively.

3.2. Sequence Annotation of Assembled Unigenes. The 66,837 unigene sequences from the assembly of the $V$. mandarinia transcriptome were used as queries for searches against the PANM, Unigene, KOG, GO, and KEGG databases by BLASTX with a cutoff $E$-value of $10^{-5}$. As shown in Table 3, overall 51,143 unigenes were matched to homologous sequences in the databases, with almost 44,922 sequences
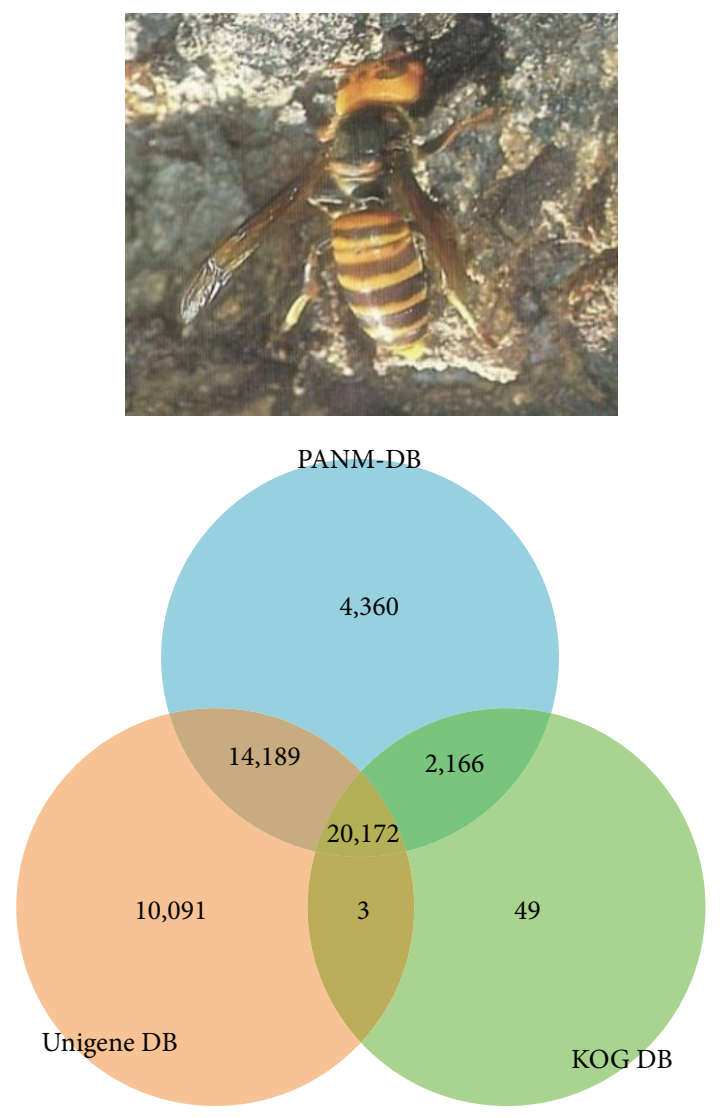

FIGURE 1: Sequence annotation of unigenes obtained from $V$. mandarinia transcriptome assembly and clustering. A three-way Venn diagram plot shows the unique and overlapped transcripts from unigene annotation hits (BLASTX; $E$-value $\leq 1 E-5$ ) against PANM-DB, Unigene, and KOG databases, respectively.

being more than $300 \mathrm{bp}$ in length. Of the 66,837 sequences, 44,455 unigenes $(66.51 \%)$ matched the Unigene database, followed by 40,887 unigenes $(61.17 \%)$ matching PANM, and 22,390 unigenes (33.5\%) matching KOG. The BLASTX results also showed matches of 15,675 (23.45\%) and 5,111 unigenes (7.65\%) to the GO and KEGG databases, respectively. In addition, our results showed that about $79.65 \%, 82.63 \%$, and $83.41 \%$ of the unigenes that were over $1000 \mathrm{bp}$ in length had BLAST matches in the KOG, GO, and KEGG databases, respectively. It is common for transcripts from transcriptome assemblies not to show homology with known proteins, as they may contain incompletely spliced introns, orphaned untranslated regions, noncoding genes, and random transcriptional noise. PANM has been found to be promising for the annotation of NGS data related to the arthropods, nematodes, and molluscs, as it stands as a filtered format of the NCBI nonredundant protein database (NCBI nr) that provides the more stringent and faster reproduction of annotation results [24].

Using a three-way Venn diagram, we studied the overlap of the annotated unigene sequences within each of the PANM, Unigene, and KOG databases (Figure 1). Interestingly, a total of 20,172 unigenes had homologous sequences 


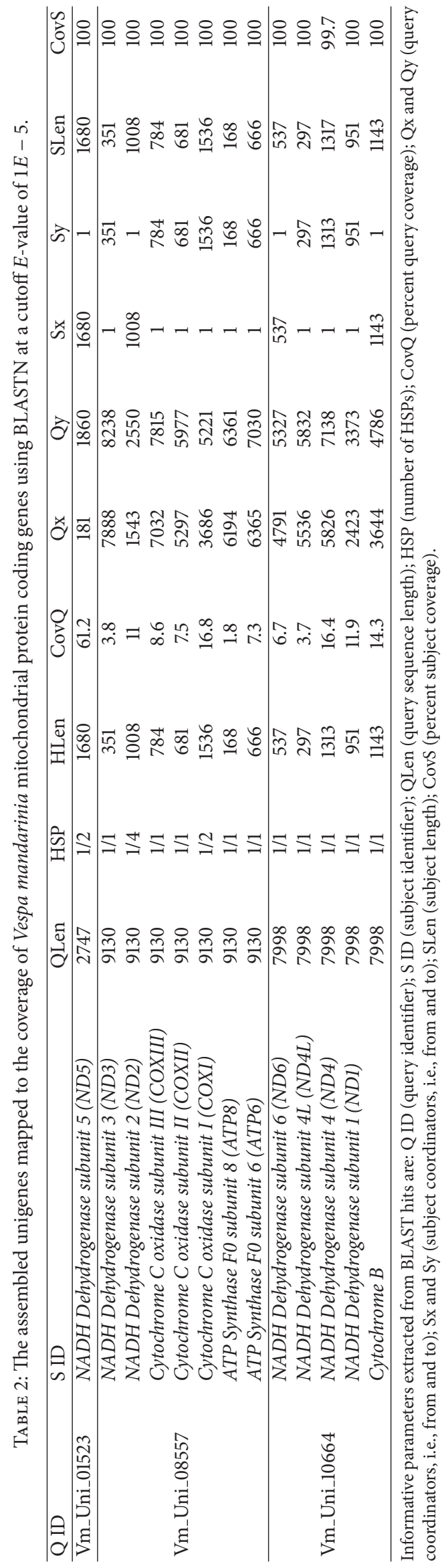




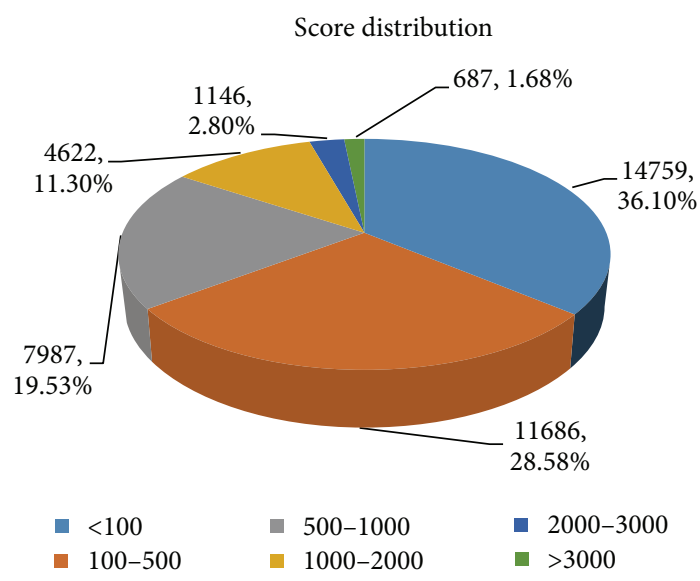

(a)

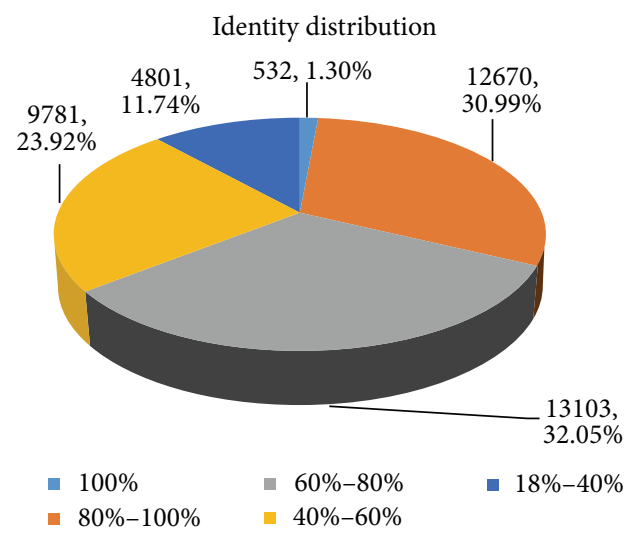

(c)

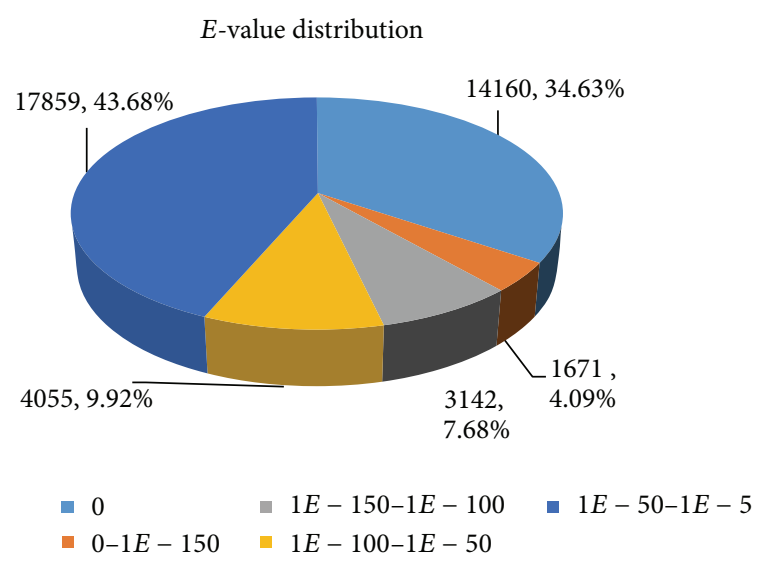

(b)

Similarity distribution



(d)

Unigene hits versus nonhits

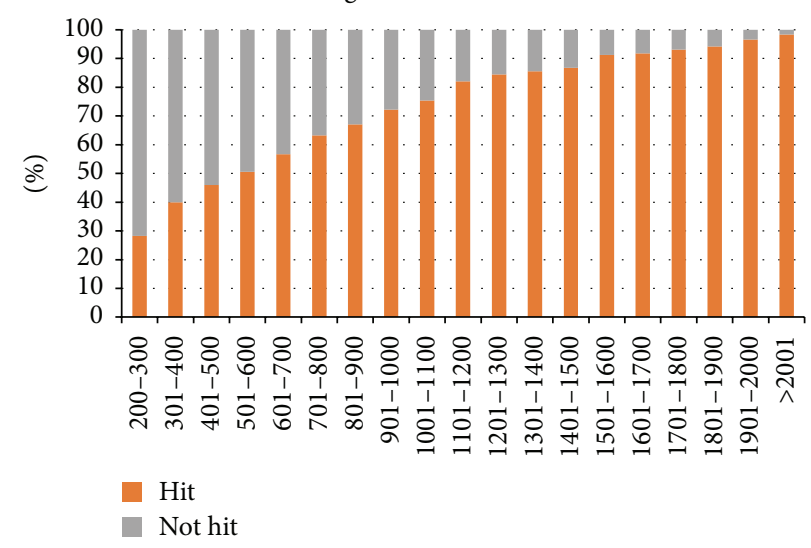

(e)

FIGURE 2: Summary of homology search of assembled unigenes of $V$. mandarinia against PANM-DB. (a) Score distribution of BLASTX hits for each unigene with a cutoff $E$-value of $1 E-5$. (b) $E$-value distribution of BLASTX hits for each unigene with a cutoff $E$-value of $1 E-5$. (c) Identity distribution of BLASTX hits for each unigene. (d) Similarity distribution of BLASTX hits for each unigene. (e) Distribution of hit and nonhit sequences as compared with the length of unigenes.

in all three databases, whereas there were overlaps of 14,189, 2,166, and 3 unigenes between the PANM and Unigene, PANM and KOG, and Unigene and KOG databases, respectively. Presumably, the unigenes that did not show any matching sequence in the databases were short transcripts lacking recognizable protein domains, or they had undergone sequence diversification.

To shed more light on the sequence hits to the PANM database, we studied the results of a homology search conducted using BLASTX (Figure 2). The PANM database 
TABLE 3: Comparison of the number of unigene annotations obtained from the different protein databases. The number of unigenes hits from locally curable PANM-DB, Unigene, KOG, GO, and KEGG databases, respectively, using BLASTX search ( $E$-value $\leq 1 E-5)$.

\begin{tabular}{lcccc}
\hline & All & $\leq 300 \mathrm{bp}$ & $300-1000 \mathrm{bp}$ & $\geq 1000 \mathrm{bp}$ \\
\hline PANM-DB & 40,887 & 3,689 & 14,531 & 22,667 \\
Unigene & 44,455 & 4,842 & 18,298 & 21,315 \\
KOG & 22,390 & 765 & 3,790 & 17,835 \\
GO & 15,675 & 625 & 2,098 & 12,952 \\
KEGG & 5,111 & 286 & 562 & 4,263 \\
All & 51,143 & 6,221 & 21,351 & 23,571 \\
\hline
\end{tabular}

was selected as it includes the protein sequences from only the protostome groups and has data processing speed 15 times faster than that of the NCBI nr database [24]. The score distribution showed that 687 unigenes $(1.68 \%)$ had scores of more than 3000, while the majority of unigenes $(14,759,36.10 \%)$ had scores of less than 100 (Figure 2(a)). The $E$-value distribution showed that 17,859 unigenes (43.68\%) showed significant homology to the deposited sequences, with $E$-values ranging from $1 E-50$ to $1 E-5$ (Figure 2(b)). The identity plot showed a more uniform distribution of the unigenes with 13,103 (32.05\%), 12,670 (30.99\%), and 9,781 unigenes (23.92\%) showing identities of $60-80 \%, 80-$ $100 \%$, and $40-60 \%$, respectively (Figure $2(\mathrm{c})$ ). The similarity distribution curve showed that the majority of unigene sequences $(22,157,54.19 \%)$ exhibited similarities of more than $80 \%$, followed by $30.33 \%$ of sequences in the range of $60-80 \%$ (Figure $2(\mathrm{~d})$ ). In addition, our results showed that unigene sequences with lengths of more than $1,500 \mathrm{bp}$ exhibited significant hits $(\geq 90 \%)$ with protein sequences in the PANM database (Figure 2(e)). It can be assumed that the protein-coding genes (especially the more highly expressed ones) give rise to longer and more full-length transcripts.

The BLASTX top-hit species distribution of the 40,887 unigenes that matched the PANM database showed sequence matches (29,456 sequences, $72.04 \%$ ) to groups of organisms belonging to the order Hymenoptera, including Megachile rotundata (4,520 sequence matches), Harpegnathos saltator $(3,343)$, Bombus terrestris $(2,262)$, Bombus impatiens $(2,031)$, and Camponotus floridanus (1,714), and few other members (Figure 3). Some distinct unigene sequences showed hits to noninsect species $(7,603$ sequences, $18.60 \%)$ and other species (3,241 sequences, $7.93 \%)$.

3.3. Functional Prediction and Classification of Assembled Unigenes. To further evaluate the putative functions of the assembled unigenes, we used the unigenes as queries for searches against the KOG database using the BLASTX program. The unigenes were classified into three major categories: "information storage and processing," "cellular processes and signaling," and "metabolism," apart from the "poorly characterized" genes (Figure 4). Out of the 22,390 unigenes annotated to the KOG database, 6,325 (28.25\%),
3,831 (17.11\%), and 3,647 (16.29\%) unigenes were classified into the "cellular processes and signaling," "information storage and processing," and "metabolism" categories, respectively. A total of 5,703 (25.47\%) unigenes were poorly characterized, and 2,884 (12.88\%) unigenes were classified as having more than one functional attribute. Overall, 25 KOG subcategories were represented by the annotated $V$. mandarinia unigenes. The "general function prediction only" $(3,844,17.17 \%)$ subcategory formed the largest group, followed by the "multi- (more than one) classified function" subcategory. Among the subcategories belonging to the three major categories, "signal transduction mechanisms" $(2,268$, $10.13 \%)$, "posttranslational modification, protein turnover and chaperones" $(1,512,6.75 \%)$, and "transcription" (1,511, $6.75 \%)$ formed the larger groups, whereas nuclear structure $(43,0.19 \%)$ and cell motility $(26,0.12 \%)$ formed the smallest.

The total unigene profile of $V$. mandarinia was also subjected to BLAST2GO analysis to define the GO term classification and KEGG pathway mapping. The GO annotated sequences were classified into three broad functional categories, namely, biological processes, cellular components, and molecular functions. The distribution of annotated unigenes in the GO functional categories and the number of unigenes ascribed to the GO terms are shown in Figures 5(a) and 5(b), respectively. A total of 13,734 unigenes were ascribed to the molecular function category, along with 9,373 and 5,427 unigenes to the biological process and cellular component categories, respectively. Among all of the unigenes ascribed to each GO functional category, 5,127, 562, and 560 unigenes were unique to the molecular function, cellular component, and biological process categories, respectively. A total of 3,433 unigenes were associated with all three functional categories. Additionally, 7,994 unigenes were associated with both biological processes and molecular functions, 4,252 with biological processes and cellular components, and 4,046 with molecular functions and cellular functions. Moreover, from the total of 15,675 unigenes, 4,849 sequences $(30.93 \%)$ were assigned to a single GO term, whereas the rest were assigned to more than one functional term. GO functional categories only suggest the grouping of a unigene in terms of its known (or predicted) function, so they are not strictly evidence of functionality. In addition, considering the evidence code distribution associated with each GO annotation (mostly inferred from electronic annotations that were computationally derived), our interpretation of the unigenes relates only to their predicted function.

Within the biological process category (Figure 6(a)), the majority of transcripts were assigned to cellular processes $(6,658$ unigenes, $26.05 \%)$, followed by metabolic processes $(5,892,23.05 \%)$ and single organismal processes $(5,245$, $20.52 \%)$. We found few transcripts that were assigned to GO term categories, such as responses to stimulus $(1,424$ unigenes, 5.57\%), signaling (1,177, 4.60\%), immune system processes $(19,0.07 \%)$, reproduction $(11,0.04 \%)$, and reproductive processes $(8,0.03 \%)$. In the molecular function category (Figure 6(b)), the majority of transcripts were assigned to binding (9,509 unigenes, $53.06 \%)$ and catalytic activity processes $(5,692,31.76 \%)$. A few transcripts were also assigned to other GO terms under categories such as antioxidant 


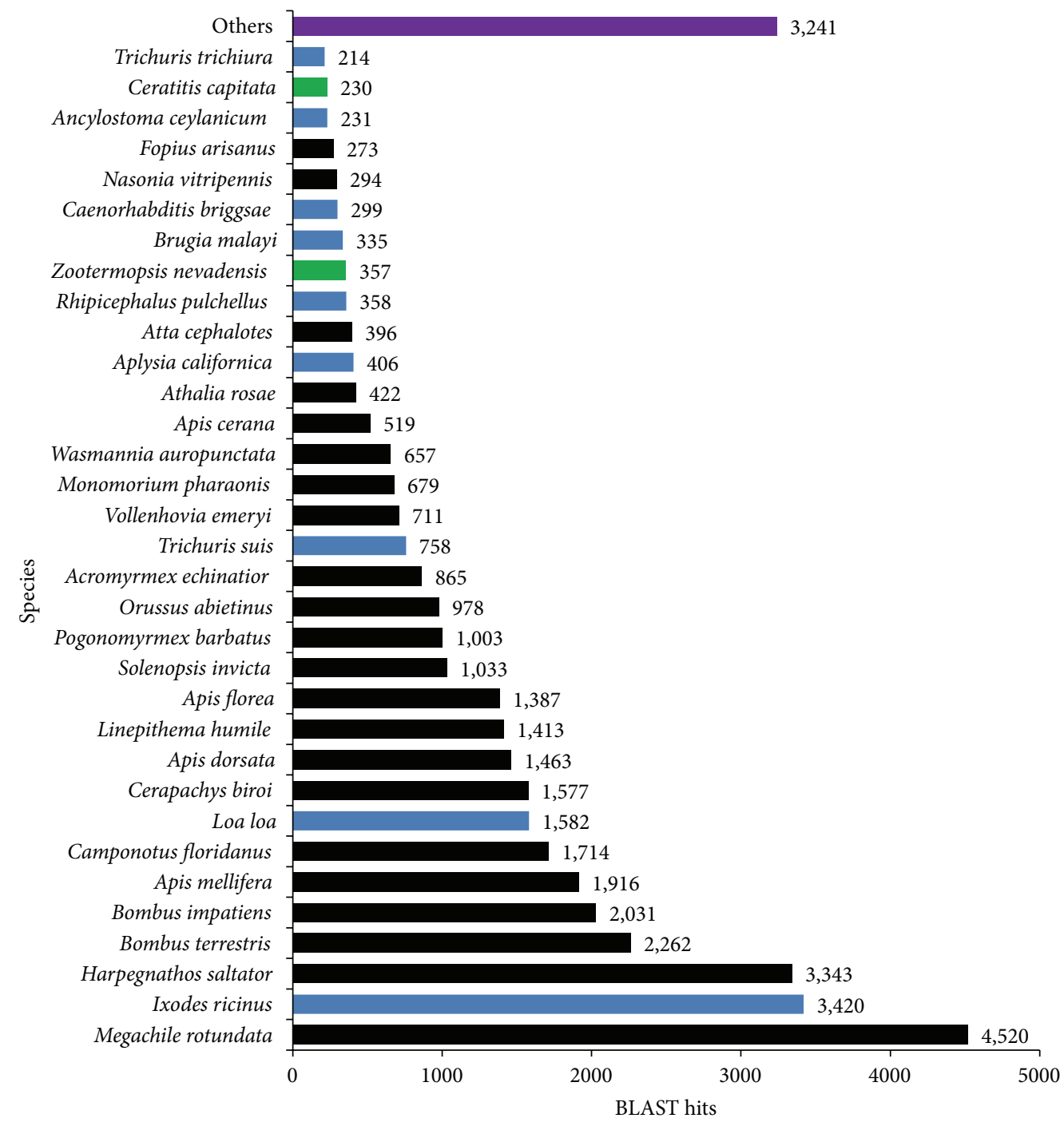

FIGURE 3: Top-hit species distribution for V. mandarinia unigenes against PANM-DB. The top-hit species belonging to class Insecta and order Hymenoptera are shaded black. The top-hit insect species not belonging to order Hymenoptera are shown by green bars. The top-hit species not belonging to class Insecta are shown by blue bars.

activity (26 unigenes, $0.15 \%$ ) and metallochaperone activity $(1,0.01 \%)$. For the transcripts representing the cellular component category (Figure 6(c)), the majority belonged to cell (2,981 unigenes, $31.44 \%)$, membrane $(2,805,29.58 \%)$, and organelle components $(2,008,21.17 \%)$. In a previous study involving a transcriptomic analysis of the Asian honey bee, Apis cerana cerana, no unigenes were ascribed to metallochaperone activity, structural molecule activity, and nucleic acid-binding transcription factor-binding activity [32]. Our study can thus be suggested to demonstrate the improved assembly of unigenes annotated to GO functional categories.

The potential involvement of the assembled V. mandarinia unigenes in biological pathways was annotated based on the KEGG database. After a search using 66,837 assembled unigenes queries in KEGG, a total of 5,111 unigenes were assigned to 115 reference canonical pathways in KEGG (Figure 7). The pathways with most unique sequences were metabolic pathways, with 4,806 unigenes (94.03\%) associated with basic metabolic functions. The predominant metabolic pathways represented by the unigenes were the amino acid metabolism $(1,591)$, metabolism of cofactors and vitamins (924), and carbohydrate metabolism pathways (521). Other pathways included the immune system (128), translation (99), and signal transduction pathways (78), representing the genetic information processing, environmental information processing, and organismal system pathways of KEGG. The putative KEGG pathways identified provide insights into the specific responses and functions involved in the biological processes of $V$. mandarinia. In the InterProScan analysis, we identified the most prominent protein domains predicted for $V$. mandarinia unigene sequences. A summary of the top InterProScan domains is provided in Table 4. The top three domains predicted are the $\mathrm{C} 2 \mathrm{H} 2$-like zinc finger domains, serine/threonine/dual specificity protein kinase domains, and RNA recognition motifs. Other common domains identified are as the WD40 repeat domains, ankyrin repeat domains, 


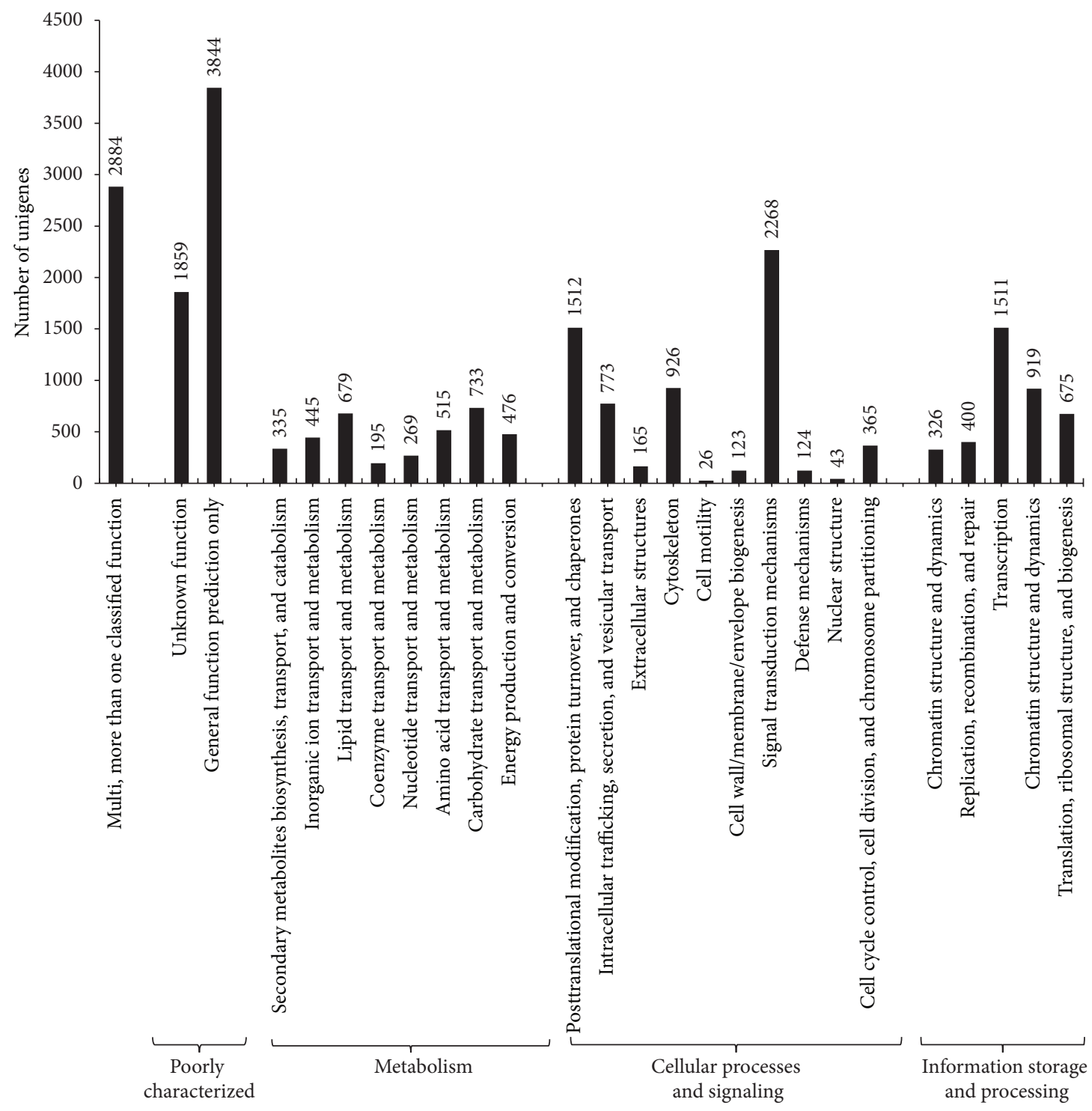

FIGURE 4: Clusters of Orthologous Groups (KOG DB) based functional analysis of V. mandarinia unigenes. The unigenes on the basis of KOG $\mathrm{DB}$ analysis are classified to metabolism, cellular processes and signaling, information storage and processing, and poorly characterized gene categories.

and the cytochrome P450 family domains. C2H2-like zinc finger domains are the most prominent DNA-binding motifs present in eukaryotic transcription factors, serving as multiple contacts in binding to DNA [33], RNA, and protein targets [34]. More specifically, they are required as parts of signal transduction, cell growth, differentiation, and development processes. Many of the binding transcription factors are proteins with a tandem array of zinc finger motifs, whereas others have a single zinc finger or multiple split pairs of $\mathrm{C} 2 \mathrm{H} 2$ zinc-fingers [35]. The RNA recognition motif domain (about 90 amino acids) is known to bind to single-stranded RNAs including heterogeneous nuclear ribonucleoproteins (hnRNPs), proteins implicated in the regulation of alternative splicing, and proteins that regulate RNA stability and translation. Such motifs were prominently identified in transcriptomic analyses of other insects [36]. The predicted WD40 repeat sequences are involved in signal transduction, cell cycle control, and apoptotic mechanisms [37]. Cytochrome P450 in insects are critical in the metabolism of xenobiotics and confer resistance to synthetic insecticides and plant allelochemicals [38, 39].

3.4. Discovery of SSR Markers. In an attempt to characterize the genome and develop new molecular markers, we discovered potential SSR markers from all 66,837 unigenes (95,657,681 bases) assembled from the V. mandarinia transcriptome. SSRs are short DNA sequences from 1 to $8 \mathrm{bp}$ in length that are built up by tandem repeats. With the advent and successful execution of NGS technologies, the routine discovery and characterization of SSRs for nonmodel organisms are on the rise [28, 31, 40,41]. SSR marker discovery and 


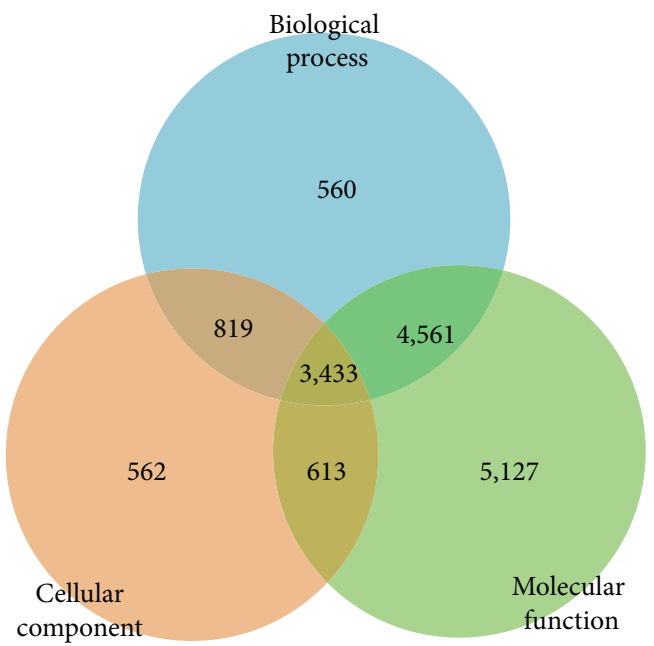

(a)

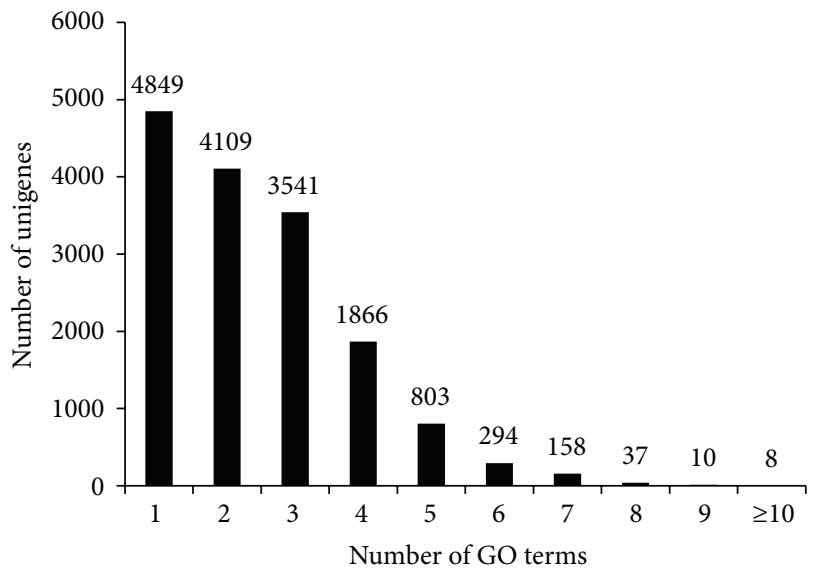

(b)

Figure 5: The GO term classification for V. mandarinia unigenes. (a) The three-way Venn diagram analysis of unigenes classified into biological process, cellular component, and molecular function categories. (b) The number of unigenes assigned to the number of GO term annotations.

TABLE 4: Summary of top 20 domains predicted in V. mandarinia unigene sequences.

\begin{tabular}{|c|c|c|c|}
\hline IPR accession & Domain short name & Domain description & Unigene \\
\hline IPR015880 & Znf_C2H2 & Zinc finger, $\mathrm{C} 2 \mathrm{H} 2$-like domain & 378 \\
\hline IPR002290 & Ser/Thr_dual-sp_kinase & Serine/threonine/dual specificity protein kinase, catalytic domain & 250 \\
\hline IPR000504 & RRM_dom & RNA recognition motif domain & 219 \\
\hline IPR001680 & WD40_repeat_dom & WD40 repeat domain & 212 \\
\hline IPR003593 & $\mathrm{AAA}+\_\mathrm{ATPase}$ & $\mathrm{AAA}+\mathrm{ATPase}$ domain & 184 \\
\hline IPR027417 & P-loop_NTPase & P-loop containing nucleoside triphosphate hydrolase domain & 178 \\
\hline IPR002110 & Ankyrin_rpt & Ankyrin repeat & 163 \\
\hline IPR011701 & MFS & Major facilitator superfamily & 148 \\
\hline IPR001478 & PDZ & PDZ domain & 144 \\
\hline IPR000008 & C2_dom & C2 domain & 119 \\
\hline IPR011989 & ARM-like & Armadillo-like helical domain & 118 \\
\hline IPR000210 & BTB/POZ_dom & $\mathrm{BTB} / \mathrm{POZ}$ domain & 116 \\
\hline IPR001452 & SH3_domain & SH3 domain & 116 \\
\hline IPR019734 & TPR_repeat & Tetratricopeptide repeat & 116 \\
\hline IPR000276 & GPCR_Rhodpsn & G protein-coupled receptor, rhodopsin-like family & 108 \\
\hline IPR001841 & Znf_RING & Zinc finger, RING-type domain & 92 \\
\hline IPR029058 & AB hydrolase & Alpha/beta hydrolase fold domain & 92 \\
\hline IPR001128 & Cyt_P450 & Cytochrome P450 family & 89 \\
\hline IPR001849 & PH_domain & Pleckstrin homology domain & 89 \\
\hline IPR003591 & Leu-rich_rpt_typical-subtyp & Leucine-rich repeat, typical subtype repeat & 89 \\
\hline
\end{tabular}

validation are strategic steps for progress in genetic diversity analysis, population genetics, conservation genomics, and association analysis [42]. The lack of genomic resources for $V$. mandarinia and the fact that the species is threatened due to the loss of its habitat indicate the importance of the discovery of SSRs via Illumina HiSeq 4000 sequencing efforts. Among the examined sequences, 62,522 sequences contained SSRs, with 56,378 sequences containing more than one of them
(Supplementary Table 2). We identified a total of 534,922 SSRs (defined as mononucleotide to decanucleotide SSRs), with 275,493 SSRs present in compound formation. After the elimination of mononucleotide and decanucleotide repeats, a total of 505,104 SSRs were screened. The frequency and distribution of 505,104 SSRs were analyzed in this study. The distribution of SSRs based on the number of repeat units is shown in Table 5 . The dinucleotide repeat motifs were 


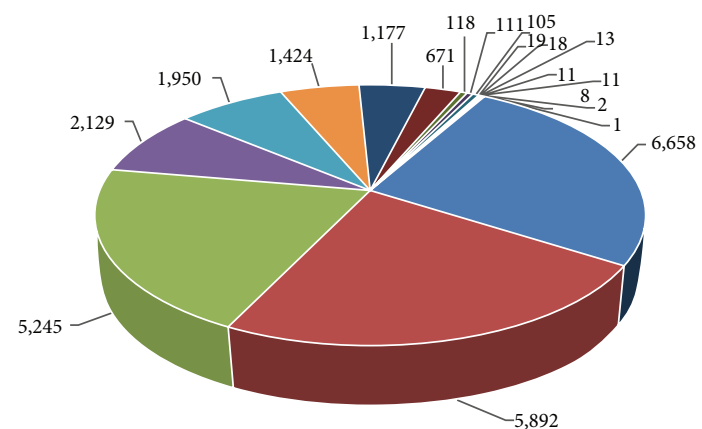

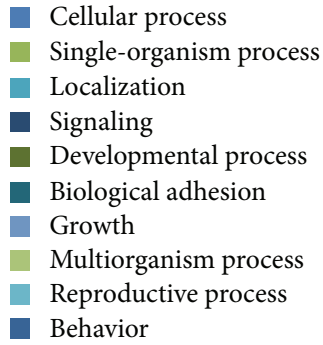

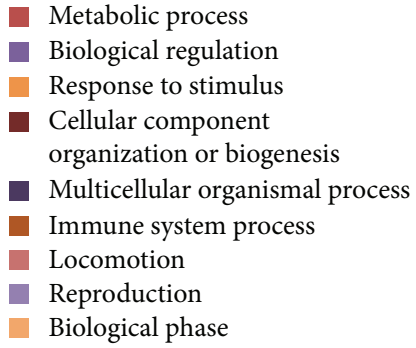

(a)

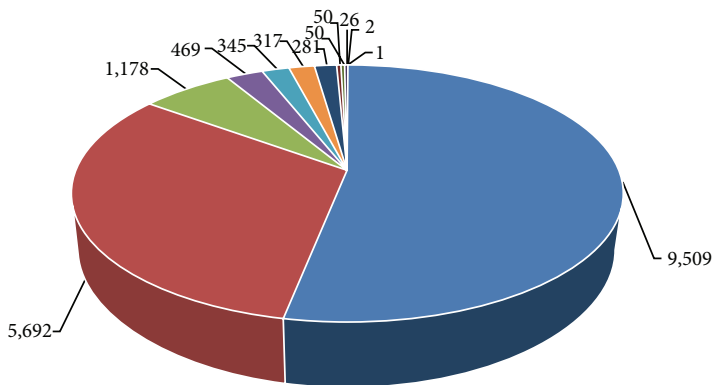

Binding

Transporter activity

Nucleic acid binding transcription factor activity

- Structural molecule activity

Electron carrier activity

Protein tag
Catalytic activity

- Molecular transducer activity

- Molecular function regulator

- Transcription factor activity, protein binding

- Antioxidant activity

Metallochaperone activity (b)

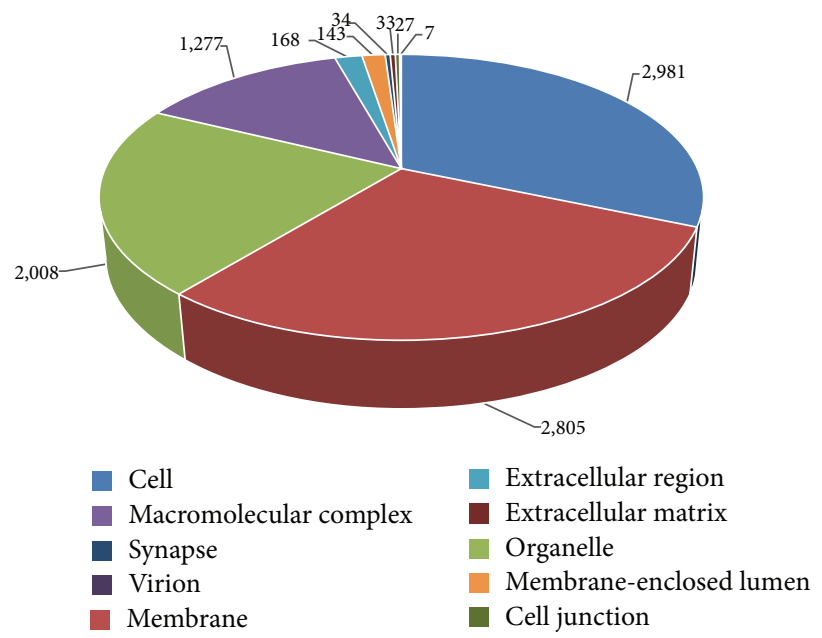

(c)

Figure 6: Gene Ontology classifications of assembled unigenes from V. mandarinia. (a) Biological processes, (b) molecular function, and (c) cellular components. The number of unigenes ascribed to each subcategory for each classification has been indicated.

the most abundant $(370,450,73.34 \%)$, followed by the trinucleotide $(105,010,20.79 \%)$ and tetranucleotide repeats $(20,182$, $4 \%)$. Penta-, hexa-, hepta-, and octanucleotide repeats were less abundant in the assembled unigene sequences. Upon further analysis of the SSR repeat numbers, we found that three tandem iterations were the most common $(372,888)$, followed by four $(59,551)$ and five random iterations $(19,793)$. SSRs with $>21$ random iterations were more common than SSRs with 12 to 20 random iterations.

The distribution of the SSRs based on motif sequence types is shown in Figure 8. Overall, 567 motif sequence types were identified, including 2 and 41 repeat types for mononucleotide and decanucleotide repeats, respectively.
From among the other repeat types, di-, tri-, tetra-, penta-, hexa-, hepta-, and octanucleotides had 4, 10, 33, 89, 197, 86, and 105 types, respectively. Notably, among the SSR types, AT/AT $(184,581,36.54 \%)$ was the most abundant motif, followed by AG/CT $(118,155,23.39 \%)$ and AC/GT $(55,624$, $11.01 \%$ ) among the dinucleotide repeats, and AAT/ATT $(32,434,6.42 \%)$ and AAG/CTT $(21,323,4.22 \%)$ among the trinucleotide repeats. The most abundant tetranucleotide and pentanucleotide repeats were AAAG/CTTT (4,714, 0.93\%) and AAAAG/CTTTT (1,568, 0.31\%), respectively. Among the other arthropods, 92 SSRs were detected for the butterfly Euphydryas editha [43], 149 for the citrus whitefly, Dialeurodes citri [44], 1,249 for Tenebrio molitor [45], and 1,100 for 


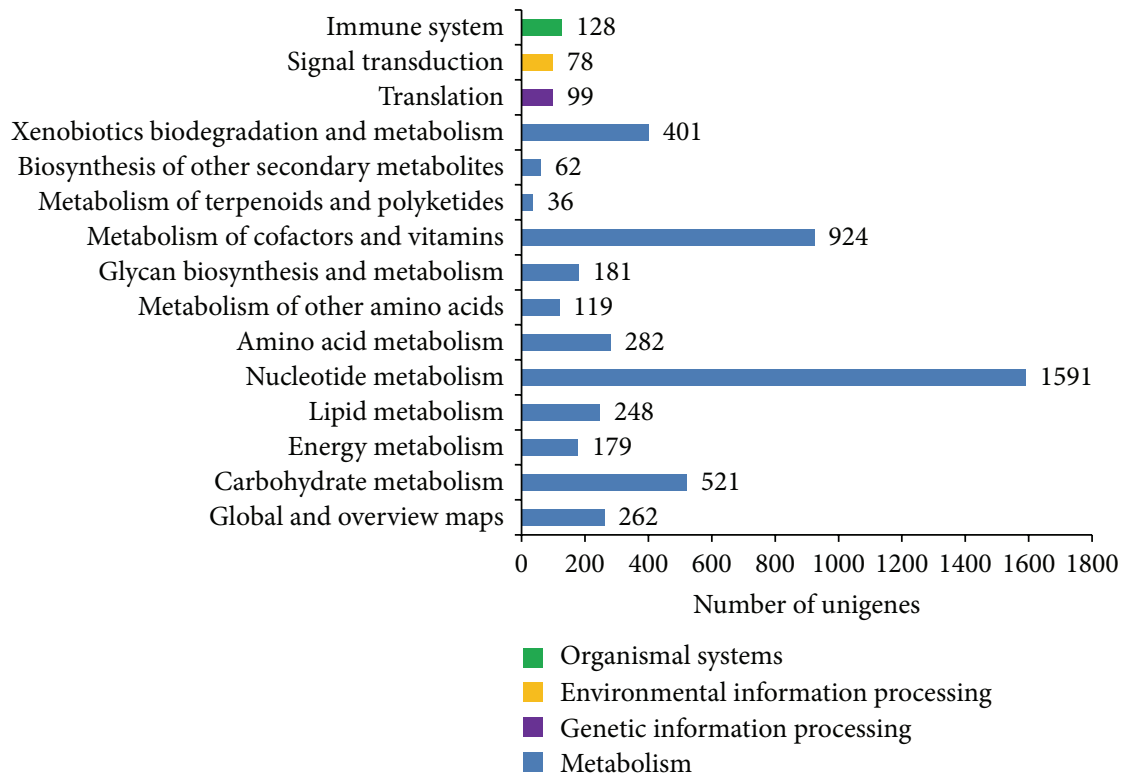

Figure 7: Kyoto Encyclopedia of Genes and Genomes (KEGG) pathway assignment for assembled unigenes from V. mandarinia. The unigenes have been categorized to metabolism, genetic information processing, environmental information processing, and organismal systems pathways.

TABLE 5: Distribution of simple sequence repeat (SSR) among the unigenes of Vespa mandarinia based on the number of repeat units.

\begin{tabular}{|c|c|c|c|c|c|c|c|c|}
\hline Repeat numbers & $\mathrm{Di}$ & Tri & Tetra & Penta & Hexa & Hepta & Octa & Total \\
\hline 3 & 275239 & 78234 & 12632 & 2814 & 3053 & 405 & 511 & 372888 \\
\hline 4 & 43886 & 10895 & 3176 & 711 & 776 & 72 & 35 & 59551 \\
\hline 5 & 12933 & 4766 & 1632 & 325 & 100 & 27 & 10 & 19793 \\
\hline 6 & 8059 & 2918 & 1394 & 48 & 87 & 18 & 5 & 12529 \\
\hline 7 & 6168 & 2096 & 224 & 79 & 75 & 13 & 4 & 8659 \\
\hline 8 & 5218 & 2082 & 325 & 35 & 32 & 1 & 0 & 7693 \\
\hline 9 & 3823 & 478 & 206 & 37 & 41 & 0 & 1 & 4586 \\
\hline 10 & 2862 & 541 & 132 & 21 & 27 & 1 & 0 & 3584 \\
\hline 11 & 2678 & 440 & 124 & 24 & 11 & 0 & 0 & 3277 \\
\hline 12 & 2175 & 347 & 72 & 15 & 4 & 0 & 0 & 2613 \\
\hline 13 & 613 & 306 & 53 & 6 & 5 & 0 & 0 & 983 \\
\hline 14 & 828 & 263 & 43 & 5 & 2 & 0 & 0 & 1141 \\
\hline 15 & 831 & 191 & 35 & 5 & 5 & 0 & 0 & 1067 \\
\hline 16 & 664 & 196 & 43 & 3 & 1 & 0 & 0 & 907 \\
\hline 17 & 659 & 171 & 22 & 1 & 2 & 0 & 0 & 855 \\
\hline 18 & 539 & 159 & 14 & 0 & 2 & 0 & 0 & 714 \\
\hline 19 & 504 & 124 & 6 & 0 & 1 & 0 & 0 & 635 \\
\hline 20 & 395 & 122 & 15 & 0 & 0 & 0 & 0 & 532 \\
\hline$>21$ & 2376 & 681 & 34 & 5 & 1 & 0 & 0 & 3097 \\
\hline Total & 370450 & 105010 & 20182 & 4134 & 4225 & 537 & 566 & 505104 \\
\hline
\end{tabular}

the stored-product pest Liposcelis entomophila [46]. Variation in the number of SSRs identified could be attributable to the selection criteria used during the analysis. Typically, SSRs sizes of two to six nucleotides in length, with minimum repeat units of six for dinucleotides, five for trinucleotides and tetranucleotides, and four for pentanucleotides and hexanucleotides, have been considered. In another study, only unigene sequences longer than $1 \mathrm{~Kb}$ with the above selection criteria were considered for SSR selection [47].

\section{Conclusions}

In this study, the complete transcriptome of the wasp $V$. mandarinia was sequenced using the Illumina HiSeq 4000 


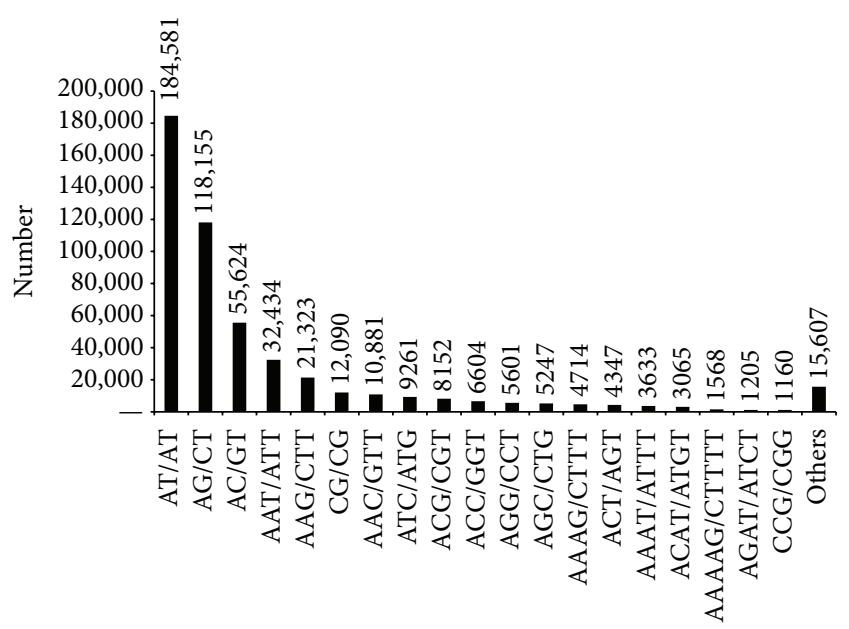

Motif

FIGURE 8: The number of simple sequence repeats (SSRs) discovered in the unigenes of $V$. mandarinia classified based on motif sequence types.

NGS platform. The sequences were assembled to meaningful unigene sequences using the de novo assembler Trinity and the clustering tool TGICL. The unigene sequences were annotated to publicly available protein sequence databases for putative functional attributes, especially their assignments to GO categories and KEGG biochemical pathways. In addition, we obtained a set of reliable SSR markers from the unigene sequences. This valuable genetic information should be useful for research on functional genes using genomic and proteomic tools. Additionally, markers can be developed to help conserve $V$. mandarinia in its preferred habitat.

\section{Conflict of Interests}

The authors declare that they have no conflict of interests.

\section{Authors' Contribution}

Bharat Bhusan Patnaik, So Young Park, and Yong Seok Lee conceived and designed the study. Tae Hun Wang, Eun Bi Park, Jong Min Chung, Dae Kwon Song, and Jae Bong Lee collected the data. Bharat Bhusan Patnaik, So Young Park, Se Won Kang, and Hee-Ju Hwang analyzed the data. Bharat Bhusan Patnaik and Se Won Kang wrote the paper. Hong Seog Park ran the sequencing experiments. Changmu Kim, Soonok Kim, and Yeon Soo Han contributed materials/analysis tools. Yong Seok Lee supervised the study. Bharat Bhusan Patnaik and So Young Park contributed equally to this work.

\section{Acknowledgment}

This work was supported by the grant entitled "The Genetic and Genomic Evaluation of Indigenous Biological Resources" funded by the National Institute of Biological Resources (NIBR201503202).

\section{References}

[1] B. J. Taylor, E. V. Nordheim, T. I. Schueller, and R. L. Jeanne, "Recruitment in swarm-founding wasps: Polybia occidentalis does not actively scent-mark carbohydrate food sources," Psyche, vol. 2011, Article ID 378576, 7 pages, 2011.

[2] M. B. Choi and O. Kwon, "Occurrence of Hymenoptera (wasps and bees) and their foraging in the southwestern part of Jirisan National Park, South Korea," Journal of Ecology and Environment, vol. 38, no. 3, pp. 367-374, 2015.

[3] M. Matsuura and S. K. Yamane, Biology of the Vespine Wasps, Springer, Berlin, Germany, 1990.

[4] T. Abe, Y. Takiguchi, M. Tamura, J. Shimura, and K. Yamazaki, "Effects of Vespa amino acid mixture (VAAM) isolated from hornet larval saliva and modified VAAM nutrients on endurance exercise in swimming mice-improvement in performance and changes of blood lactate and glucose," Japanese Journal of Physical Fitness and Sports Medicine, vol. 44, no. 2, pp. 225-238, 1995.

[5] H. Sasai, T. Matsuo, M. Fujita, M. Saito, and K. Tanaka, "Effects of regular exercise combined with ingestion of vespa amino acid mixture on aerobic fitness and cardiovascular disease risk factors in sedentary older women: a preliminary study," Geriatrics and Gerontology International, vol. 11, no. 1, pp. 2431, 2011.

[6] Y. Yanagawa, K. Morita, T. Suguira, and Y. Okada, "Cutaneous hemorrhage or necrosis findings after Vespa mandarinia (wasp) stings may predict the occurrence of multiple organ injury: a case report and review of literature," Clinical Toxicology, vol. 45, no. 7, pp. 803-807, 2007.

[7] D. C. de Graaf, M. Aerts, E. Danneels, and B. Devreese, "Bee, wasp and ant venomics pave the way for a component-resolved diagnosis of sting allergy," Journal of Proteomics, vol. 72, no. 2, pp. 145-154, 2009.

[8] L. Chen, W. Chen, H. Yang, and R. Lai, "A novel bioactive peptide from wasp venom," Journal of Venom Research, vol. 1, pp. 43-47, 2010.

[9] H. Kishimura, T. Yasuhara, H. Yoshida, and T. Nakajima, "Vespakinin-M, a novel bradykinin analogue containing hydroxyproline, in the venom of Vespa mandarinia Smith," Chemical and Pharmaceutical Bulletin, vol. 24, no. 11, pp. 2896-2897, 1976.

[10] Y. Hirai, T. Yasuhara, H. Yoshida, and T. Nakajima, "A new mast cell degranulating peptide, mastoparan- $\mathrm{M}$, in the venom of the hornet Vespa mandarinia," Biomedical Research, vol. 2, no. 4, pp. 447-449, 1981.

[11] C. M. Grozinger, Y. Fan, S. E. R. Hoover, and M. L. Winston, "Genome-wide analysis reveals differences in brain gene expression patterns associated with caste and reproductive status in honey bees (Apis mellifera)," Molecular Ecology, vol. 16, no. 22, pp. 4837-4848, 2007.

[12] B. M. Sadd, S. M. Barribeau, G. Bloch et al., "The genomes of two key bumblebee species with primitive eusocial organization," Genome Biology, vol. 16, article 76, 2015.

[13] C. C. Judice, M. F. Carazzole, F. Festa, M. C. Sogayar, K. Hartfelder, and G. A. G. Pereira, "Gene expression profiles underlying alternative caste phenotypes in a highly eusocial bee, Melipona quadrifasciata," Insect Molecular Biology, vol. 15, no. 1, pp. 33-44, 2006.

[14] P. G. Ferreira, S. Patalano, R. Chauhan et al., "Transcriptome analyses of primitively eusocial wasps reveal novel insights 
into the evolution of sociality and the origin of alternative phenotypes," Genome Biology, vol. 14, no. 2, article R20, 2013.

[15] A. L. Toth, K. Varala, M. T. Henshaw, S. L. Rodriguez-Zas, M. E. Hudson, and G. E. Robinson, "Brain transcriptomic analysis in paper wasps identifies genes associated with behaviour across social insect lineages," Proceedings of Biological Sciences, vol. 277, no. 1691, pp. 2139-2148, 2010.

[16] Y. Wang, S. D. Kocher, T. A. Linksvayer, C. M. Grozinger, R. E. Page Jr., and G. V. Amdam, "Regulation of behaviorally associated gene networks in worker honey bee ovaries," Journal of Experimental Biology, vol. 215, no. 1, pp. 124-134, 2012.

[17] A. J. Berens, J. H. Hunt, and A. L. Toth, "Comparative transcriptomics of convergent evolution: different genes but conserved pathways underlie caste phenotypes across lineages of eusocial insects," Molecular Biology and Evolution, vol. 32, no. 3, pp. 690703, 2015.

[18] A. Bräutigam, T. Mullick, S. Schliesky, and A. P. M. Weber, "Critical assessment of assembly strategies for non-model species mRNA-Seq data and application of next-generation sequencing to the comparison of $\mathrm{C}_{3}$ and $\mathrm{C}_{4}$ species," Journal of Experimental Botany, vol. 62, no. 9, pp. 3093-3102, 2011.

[19] Y. Qi, Z. Teng, L. Gao et al., "Transcriptome analysis of an endoparasitoid wasp Cotesia chilonis (Hymenoptera: Braconidae) reveals genes involved in successful parasitism," Archives of Insect Biochemistry and Physiology, vol. 88, no. 4, pp. 203-221, 2015.

[20] W. Bouzid, M. Verdenaud, C. Klopp, F. Ducancel, C. Noirot, and A. Vetillard, "De novo sequencing and transcriptome analysis for Tetramorium bicarinatum: a comprehensive venom gland transcriptome analysis from an ant species," BMC Genomics, vol. 15, article 987, 2014.

[21] B. M. Jones, W. T. Wcislo, and G. E. Robinson, "Developmental transcriptome for a facultatively eusocial bee, Megalopta genalis," G3: Genes, Genomes, Genetics Mission, vol. 5, no. 10, pp. 2127-2135, 2015.

[22] M. G. Grabherr, B. J. Haas, M. Yassour et al., "Full-length transcriptome assembly from RNA-Seq data without a reference genome," Nature Biotechnology, vol. 29, no. 7, pp. 644-652, 2011.

[23] G. Pertea, X. Huang, F. Liang et al., "TIGR gene indices clustering tools (TGICL): a software system for fast clustering of large EST datasets," Bioinformatics, vol. 19, no. 5, pp. 651-652, 2003.

[24] S. W. Kang, B. B. Patnaik, H. J. Hwang et al., "PANM-DB (Protostome DB) for the annotation of NGS data of mollusks," The Korean Journal of Malacology, vol. 31, pp. 243-247, 2015.

[25] M. Kanehisa, S. Goto, S. Kawashima, Y. Okuno, and M. Hattori, "The KEGG resource for deciphering the genome," Nucleic Acids Research, vol. 32, supplement 1, pp. D277-D280, 2004.

[26] A. Conesa, S. Götz, J. M. García-Gómez, J. Terol, M. Talón, and M. Robles, "BLAST2GO: a universal tool for annotation, visualization and analysis in functional genomics research," Bioinformatics, vol. 21, no. 18, pp. 3674-3676, 2005.

[27] B. Feldmeyer, C. W. Wheat, N. Krezdorn, B. Rotter, and M. Pfenninger, "Short read Illumina data for the de novo assembly of a non-model snail species transcriptome (Radix balthica, Basommatophora, Pulmonata), and a comparison of assembler performance," BMC Genomics, vol. 12, article 317, 2011.

[28] R. Che, Y. Sun, R. Wang, and T. Xu, "Transcriptomic analysis of endangered Chinese salamander: identification of immune, sex and reproduction-related genes and genetic markers," PLoS ONE, vol. 9, no. 1, Article ID e87940, 2014.
[29] S. M. Gross, S. Pathak, L. C. Watson, and G. P. Schroth, A First Look at RNA-Seq Data on the HiSeq 4000 System, Illumina Applications Group, 2015.

[30] Z. Xia, H. Xu, J. Zhai et al., "RNA-Seq analysis and de novo transcriptome assembly of Hevea brasiliensis," Plant Molecular Biology, vol. 77, no. 3, pp. 299-308, 2011.

[31] L. Wei, S. Li, S. Liu et al., "Transcriptome analysis of Houttuynia cordata Thunb. by illumina paired-end RNA sequencing and SSR marker discovery," PLoS ONE, vol. 9, no. 1, Article ID e84105, 2014.

[32] Z. L. Wang, T. T. Liu, Z. Y. Huang, X. B. Wu, W. Y. Yan, and Z. J. Zeng, "Transcriptome analysis of the Asian honey bee Apis cerana cerana," PLoS ONE, vol. 7, no. 10, Article ID e47954, 2012.

[33] S. A. Wolfe, L. Nekludova, and C. O. Pabo, "DNA recognition by $\mathrm{Cys}_{2} \mathrm{His}_{2}$ zinc finger proteins," Annual Review of Biophysics and Biomolecular Structure, vol. 29, pp. 183-212, 2000.

[34] K. J. Brayer and D. J. Segal, "Keep your fingers off my DNA: protein-protein interactions mediated by $\mathrm{C} 2 \mathrm{H} 2$ zinc finger domains," Cell Biochemistry and Biophysics, vol. 50, no. 3, pp. 111-131, 2008.

[35] A. Seetharam, Y. Bai, and G. W. Stuart, "A survey of well conserved families of $\mathrm{C} 2 \mathrm{H} 2$ zinc-finger genes in Daphnia," BMC Genomics, vol. 11, article 276, 2010.

[36] Y. Matsumoto, Y. Suetsugu, M. Nakamura, and M. Hattori, "Transcriptome analysis of the salivary glands of Nephotettix cincticeps (Uhler)," Journal of Insect Physiology, vol. 71, pp. 170176, 2014.

[37] D. Li and R. Roberts, "WD-repeat proteins: structure characteristics, biological function, and their involvement in human diseases," Cellular and Molecular Life Sciences, vol. 58, no. 14, pp. 2085-2097, 2001.

[38] X. Bai, P. Mamidala, S. P. Rajarapu, S. C. Jones, and O. Mittapalli, "Transcriptomics of the bed bug (Cimex lectularius)," PLoS ONE, vol. 6, no. 1, Article ID e16336, 2011.

[39] H.-P. Lu, M. Edwards, Q.-Z. Wang et al., "Expression of cytochrome P450 CYP81A6 in rice: tissue specificity, protein subcellular localization, and response to herbicide application," Journal of Zhejiang University: Science B, vol. 16, no. 2, pp. 113122, 2015.

[40] J. E. Zalapa, H. Cuevas, H. Zhu et al., "Using next-generation sequencing approaches to isolate simple sequence repeat (SSR) loci in the plant sciences," American Journal of Botany, vol. 99, no. 2, pp. 193-208, 2012.

[41] M. M. López-Uribe, C. K. Santiago, S. M. Bogdanowicz, and B. N. Danforth, "Discovery and characterization of microsatellites for the solitary bee Colletes inaequalis using Sanger and 454 pyrosequencing," Apidologie, vol. 44, no. 2, pp. 163-172, 2013.

[42] Z.-G. Wu, W. Jiang, N. Mantri, X.-Q. Bao, S.-L. Chen, and Z.M. Tao, "Transcriptomic analysis reveals flavonoid biosynthesis regulation and simple sequence repeats in yam (Dioscorea alata L.) tubers," BMC Genomics, vol. 16, article 346, 2015.

[43] A. S. Mikheyev, T. Vo, B. Wee, M. C. Singer, and C. Parmesan, "Rapid microsatellite isolation from a butterfly by De novo transcriptome sequencing: performance and a comparison with AFLP-derived distances," PLoS ONE, vol. 5, no. 6, Article ID e11212, 2010.

[44] E.-H. Chen, D.-D. Wei, G.-M. Shen, G.-R. Yuan, P.-P. Bai, and J.-J. Wang, "De novo characterization of the Dialeurodes citri transcriptome: mining genes involved in stress resistance and simple sequence repeats (SSRs) discovery," Insect Molecular Biology, vol. 23, no. 1, pp. 52-66, 2014. 
[45] J.-Y. Zhu, G.-X. Wu, and B. Yang, "High-throughput discovery of SSR genetic markers in the yellow mealworm beetle, Tenebrio molitor (Coleoptera: Tenebrionidae), from its transcriptome database," Acta Entomologica Sinica, vol. 56, pp. 724-728, 2013.

[46] D.-D. Wei, E.-H. Chen, T.-B. Ding, S.-C. Chen, W. Dou, and J.-J. Wang, "De novo assembly, gene annotation, and marker discovery in stored-product pest Liposcelis entomophila (Enderlein) using transcriptome sequences," PLoS ONE, vol. 8, no. 11, Article ID e80046, 2013.

[47] Y.-J. Zhang, Y. Hao, F. Si et al., "The de novo transcriptome and its analysis in the worldwide vegetable pest, Delia antiqua (Diptera: Anthomyiidae)," G3, vol. 4, no. 5, pp. 851-859, 2014. 

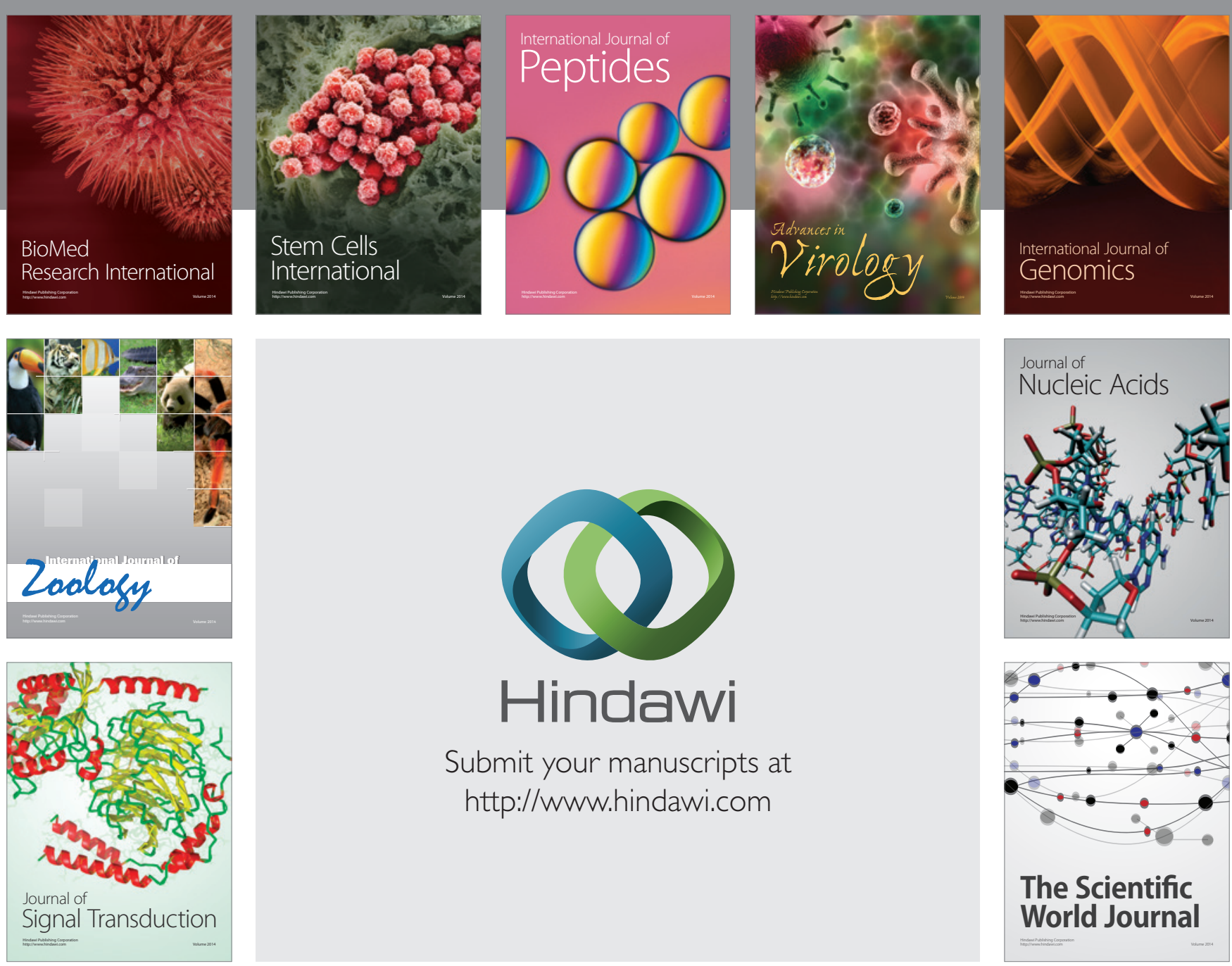

Submit your manuscripts at

http://www.hindawi.com
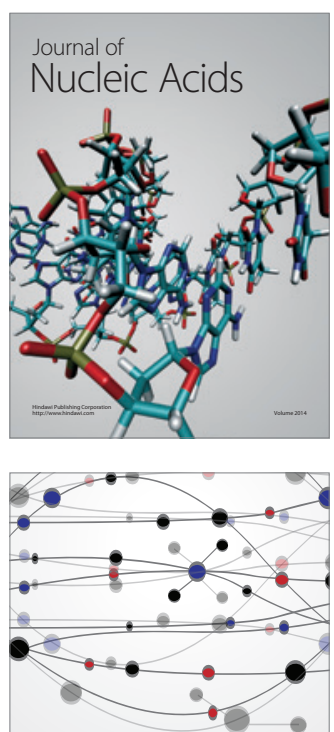

The Scientific World Journal
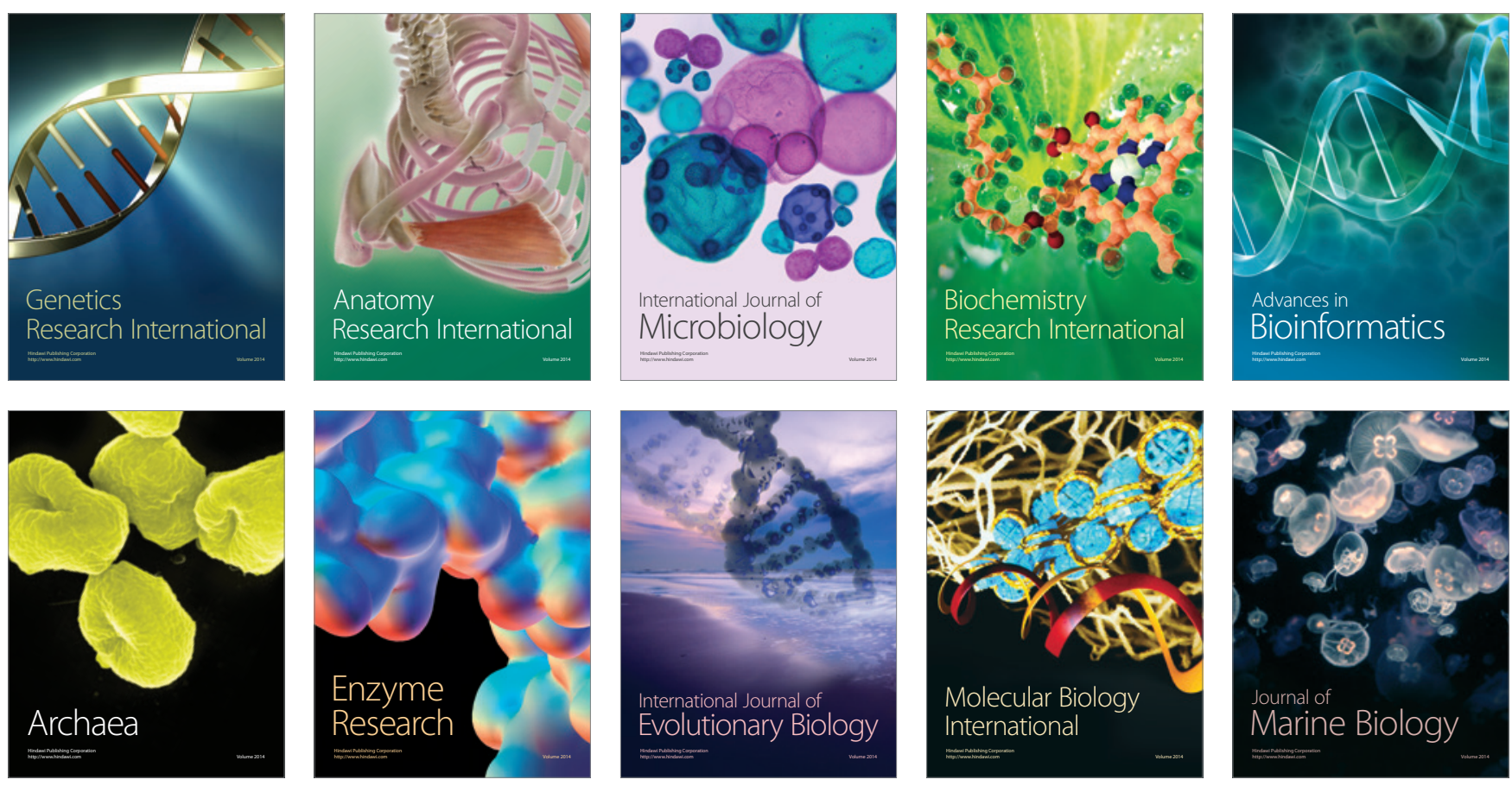